\title{
Variational matrix product ansatz for nonuniform dynamics in the thermodynamic limit
}

\author{
Ashley Milsted, ${ }^{1, *}$ Jutho Haegeman, ${ }^{2}$ Tobias J. Osborne, ${ }^{1}$ and Frank Verstraete ${ }^{2}$ \\ ${ }^{1}$ Leibniz Universität Hannover, Institute of Theoretical Physics, Appelstrasse 2, D-30167 Hannover, Germany \\ ${ }^{2}$ Vienna Center for Quantum Science and Technology, Faculty of Physics, University of Vienna, Boltzmanngasse 5, A-1090 Wien, Austria
}

(Received 20 June 2013; revised manuscript received 30 September 2013; published 14 October 2013)

\begin{abstract}
We describe how to implement the time-dependent variational principle for matrix product states in the thermodynamic limit for nonuniform lattice systems. This is achieved by confining the nonuniformity to a (dynamically expandable) finite region with fixed boundary conditions. The suppression of nonphysical quasiparticle reflections from the boundary of the nonuniform region is also discussed. Using this algorithm we study the dynamics of localized excitations in infinite systems, which we illustrate in the case of the spin-1 antiferromagnetic Heisenberg model and the $\phi^{4}$ model.
\end{abstract}

DOI: 10.1103/PhysRevB.88.155116

PACS number(s): 75.10.Jm, 05.10.Cc, 02.70.-c, 75.40.Gb

Douglas Adams (nearly) put it best: "[Hilbert] space is big... You just won't believe how vastly hugely mindbogglingly big it is. I mean, you may think it's a long way down the road to the chemist, but that's just peanuts compared to [Hilbert] space." Given said space's exponential growth with the size of a many-particle system, it is a little astounding that general techniques exist to allow efficient numerical calculations in a wide range of physically interesting cases. This is possible because physically relevant states have limited entanglement. ${ }^{1-3}$ This observation may be exploited to obtain an efficient parametrization of this physical corner of Hilbert space.

The class of matrix product states (MPSs) ${ }^{4}$ represents, in one dimension, a good parametrization of the physical corner. This is amply demonstrated by the unparalleled success of the density matrix renormalization group (DMRG), ${ }^{5}$ which can be viewed as a variational method when formulated in the MPS language. ${ }^{6}$ The MPS class has served as the basis for many exciting generalizations, including the study of nonequilibrium dynamics $^{7}$ and higher-dimensional systems. ${ }^{8}$ More recently, Haegeman et al. have implemented the time-dependent variational principle (TDVP—see boxout) for MPSs, ${ }^{9}$ providing a locally optimal (in time) framework for simulating dynamics, including finding ground states via imaginary time evolution, and an ansatz for studying excitations of one-dimensional lattice systems.

The simulation of infinite quantum spin systems has mostly been confined to the translation invariant setting (usually by restricting states to subsets of MPSs that are either fully translation invariant or invariant under translations by $k>1$ sites $\left.^{10}\right)$. However, the ability to explore locally nonuniform states on an infinite lattice is particularly attractive for studying the dynamics, e.g., scattering, of localized excitations in large systems. For example, this would provide a realistic setting in which to study quantum field excitations. There has been some prior work in this direction, building on previous light-cone results, ${ }^{3,11,12}$ where the dynamics of a local disturbance is (partially) studied in the Heisenberg picture. These approaches can become expensive for systems with large local spin dimensions (such as those appearing in lattice field theory). Another direction that has been suggested, ${ }^{13}$ is to work completely in the Schrödinger picture with infinite uniform MPSs and to add a finite nonuniform region.

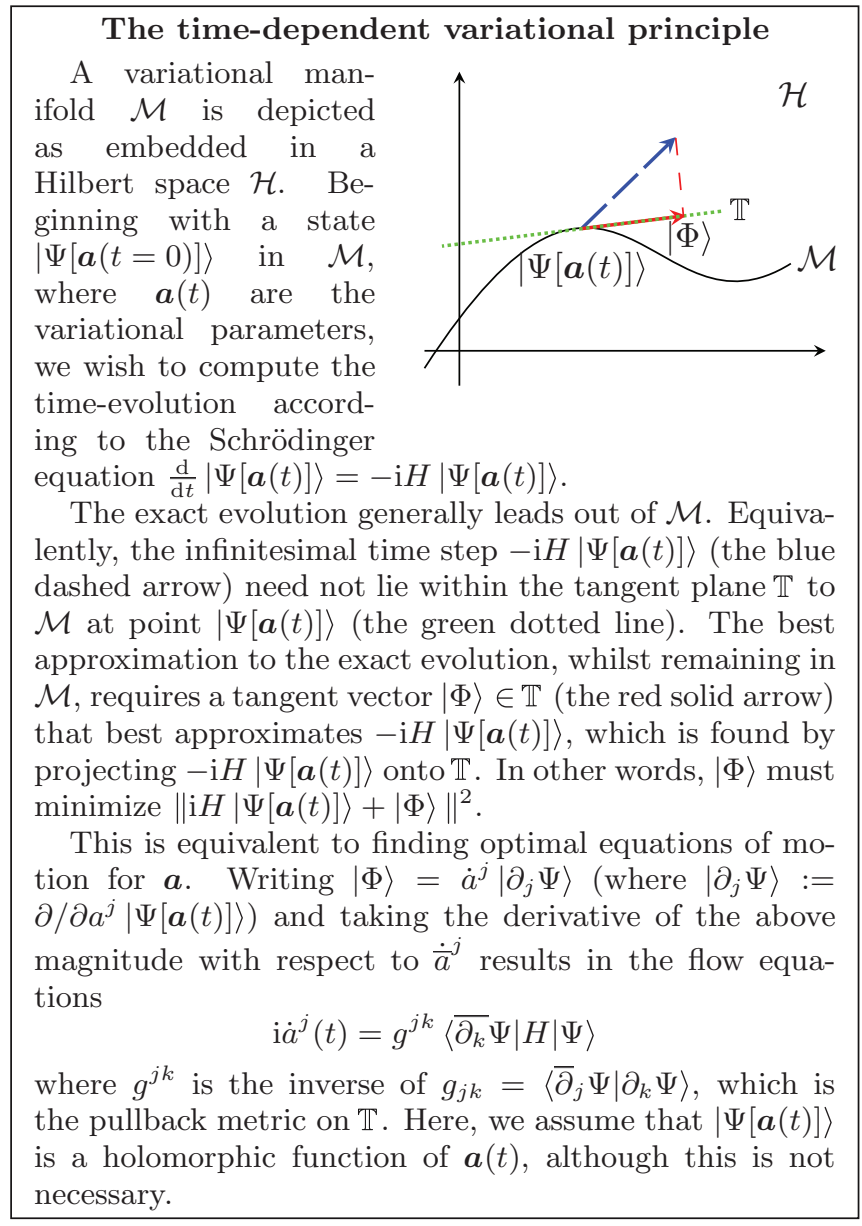

In this work we explore the locally optimal implementation of the TDVP for uniform MPSs with a dynamically expandable nonuniform segment. We derive the equations of motion for the variational parameters using a particular choice of gauge fixing which allows us to integrate the variational dynamics with a complexity that scales as $d|t| D^{3} N$, where $N$ is the length of the nonuniform piece (the number of sites), $|t|$ is the desired integration time, $d$ is the local spin dimension, and $D$ is the bond dimension. Even though the ends of the nonuniform 
region can move, there may be some backscattering due to boundary effects; we describe how to compensate for these with the addition of an optical potential term. These methods are illustrated in the case of local excitations of the spin-1 antiferromagnetic Heisenberg model and for particles in $\phi^{4}$ theory.

We assume throughout that our Hamiltonian $H$ contains only nearest-neighbor terms. It is decomposed as $H=H^{\text {uni }}+$ $H^{\text {loc }}$, where $H^{\text {uni }}=\sum_{n=-\infty}^{\infty} h_{n, n+1}^{\text {uni }}$ with $h_{n, n+1}^{\text {uni }} \cong h_{m, m+1}^{\text {uni }}$, $\forall n, m$, and $H^{\text {loc }}=\sum_{n=1}^{N-1} h_{n, n+1}^{\text {loc }}$ with $[1, N]$ representing a contiguous region of the lattice and $h_{n, n+1}^{\text {loc }} \equiv 0$ for $n<1, n \geqslant$ $N$, allowing us to also write $H=\sum_{n} h_{n, n+1}=\sum_{n}\left[h_{n, n+1}^{\text {uni }}+\right.$ $\left.h_{n, n+1}^{\text {loc }}\right]$. We consider two cases in particular: First, a nontrivial $h^{\text {loc }}$ leads to a locally nonuniform ground state, which can be found using imaginary time evolution via our algorithm. Second, given a purely uniform Hamiltonian $\left(h^{\text {loc }}=0\right)$ and an initial state that differs only locally (in a region $[1, N]$ ) from an eigenstate of $H^{\text {uni }}$, our algorithm can be used to simulate the resulting locally nontrivial dynamics.

To capture a locally nonuniform state using MPS, we define a class of "sandwich" states (sMPSs), based on uniform MPSs, using two $d \times D \times D$ tensors $A_{L}$ and $A_{R}$ describing the (asymptotic) state on either side of the nonuniform region $[1, N]$, which is parametrized by $N$ further tensors. An sMPS can be written as

$$
\begin{aligned}
|\Psi[A]\rangle= & \sum_{\{s\}=1}^{d} v_{L}^{\dagger}\left[\prod_{i=-\infty}^{0} A_{L}^{s_{i}}\right] \\
& \times A_{1}^{s_{1}} \cdots A_{N}^{s_{N}}\left[\prod_{j=N+1}^{\infty} A_{R}^{s_{j}}\right] v_{R}|s\rangle,
\end{aligned}
$$

where $|s\rangle=\left|\cdots s_{1} \cdots s_{N} \cdots\right\rangle$ and $A_{X}^{s} \in M_{D}(\mathbb{C})$ (where $X=$ $L, R,[1, N])$. Taking $A_{L}=A_{1}=\cdots=A_{N}=A_{R}$ gives a completely uniform state. The vectors $v_{L / R}$ are, as with uniform MPSs, ${ }^{9}$ generically irrelevant to the TDVP algorithm and are not further specified. In principle, the dimensions of $A_{X}^{s}$ are subject only to the constraints of the matrix product, which can become important when maximizing numerical efficiency. However, for reasons of notational simplicity, we assume uniform dimensions here.

$A_{L / R}$ represent the left and right asymptotic states: The reduced density matrix $\rho_{[n, m]}\left(A_{L}, A_{R}, A_{1 \ldots N}\right)$ of a piece of the lattice in the left or right region $n, m<1$ or $n, m>N$ tends to that of the uniform MPS $\rho_{[n, m]}\left(A_{L / R}\right)$ as the distance from the nonuniform region increases. Since $A_{L / R}$ represent infinite "bulk" regions of the lattice, their dynamics should not be affected by nonuniformities in the $[1, N]$ region, which spread at a finite speed. Furthermore, if the left and right asymptotic states are eigenstates of $H^{\text {uni }}$, they are left completely unchanged by time evolution. Assuming this, we restrict the variational parameters to the tensors $A_{1} \cdots A_{N}$ and treat $A_{L / R}$ as boundary conditions. $A_{L / R}$ can be obtained for the ground state of $H^{\text {uni }}$ using the existing TDVP algorithm for uniform MPSs. ${ }^{9}$ To accurately capture states with a nonuniform region $[1, N]$ in this way, $N$ should be sufficiently large so that the asymptotic states are already reached at the left and right boundaries with the bulk.
The tensor network formed by the matrices $A$ can be visualized as

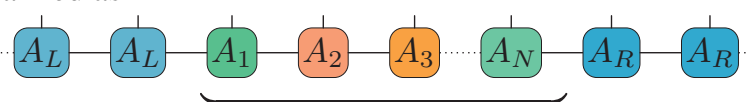

with the nonuniform region marked in the center and the physical indices pointing upwards. Expectation values of local operators can be calculated efficiently in terms of operators $E_{B}^{A} \equiv \sum_{s}^{d} A^{s} \otimes \bar{B}^{s}$, with the "transfer operators" $E_{n} \equiv E_{A_{n}}^{A_{n}}$. For example, the expectation value of an operator $h$ that acts nontrivially on a pair of neighboring sites can be written as

$$
\left\langle h_{n, n+1}\right\rangle=\left\langle v_{L}\left|\left[\prod_{k=-\infty}^{n-1} E_{n}\right] E_{n}^{h}\left[\prod_{k=n+2}^{\infty} E_{n}\right]\right| v_{R}\right\rangle,
$$

with $\quad\left\langle v_{L}\right|=v_{L}^{\dagger} \otimes \bar{v}_{L}^{\dagger} \quad$ and $\quad\left|v_{R}\right\rangle=v_{R} \otimes \bar{v}_{R} \quad$ as $\quad$ well as $E_{n<1} \equiv E_{L}$ and $E_{n>N} \equiv E_{R}$ and where $E_{n}^{h}=$ $\sum_{s, t, u, v}^{d}\langle s, t|h| u, v\rangle A_{n}^{u} A_{n+1}^{v} \otimes \overline{A_{n}^{s} A_{n+1}^{t}}$.

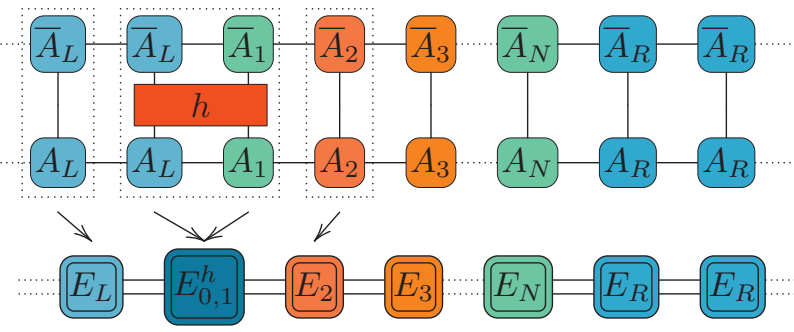

Expressions for expectation values and for the norm of the state contain parts " $\left\langle v_{L}\right|\left(E_{L}\right)$ " and " $\left(E_{R}\right)^{\infty}\left|v_{R}\right\rangle$ " that need not be well defined, depending on the properties of $E_{L}$ and $E_{R}$. To make these quantities finite, we must require that $E_{L / R}$ have a spectral radius equal to 1 . To ensure that $v_{L}$ and $v_{R}$ remain irrelevant in calculations of bulk properties, we further demand that there is a single, nondegenerate (so that $A_{L / R}^{S}$ are not block diagonalizable) eigenvalue of largest magnitude that is equal to 1, with all other eigenvalues having magnitude strictly less that $1 .^{14}$ The left and right eigenvectors corresponding to this eigenvalue, which are thus the unique left and right fixed points of $E_{L / R}$, we name $\left\langle l_{L / R}\right|$ and $\left|r_{L / R}\right\rangle$, normalizing them such that $\left\langle l_{L / R} \mid r_{L / R}\right\rangle=1$. We can then write $\langle x|\left(E_{L}\right)^{\infty} \propto\left\langle l_{L}\right|$ and $\left(E_{R}\right)^{\infty}|x\rangle \propto\left|r_{R}\right\rangle$, where $|x\rangle$ is some vector that is not orthogonal to $\left\langle l_{L}\right|$ or $\left|r_{R}\right\rangle$.

We now have a slightly simpler form for (1): $\left\langle h_{n, n+1}\right\rangle=$ $\left\langle l_{L}\left|\left[\prod_{k=1}^{n-1} E_{n}\right] E_{n}^{h}\left[\prod_{k=n+2}^{N} E_{n}\right]\right| r_{R}\right\rangle$. To further improve the notation, we define $\left\langle l_{n \geqslant 1}\right|=\left\langle l_{n-1}\right| E_{n}$ and $\left|r_{n<N}\right\rangle=E_{n+1}\left|r_{n+1}\right\rangle$, identifying $\left\langle l_{n<1}\right| \equiv\left\langle l_{L}\right|$ and $\left|r_{n \geqslant N}\right\rangle \equiv\left|r_{R}\right\rangle$ (we will also use $A_{n>N} \equiv A_{R}$ and $\left.A_{n<1} \equiv A_{L}\right)$. We then have $\left\langle h_{n, n+1}\right\rangle=$ $\left\langle l_{n-1}\left|E_{n}^{h}\right| r_{n+1}\right\rangle$ :

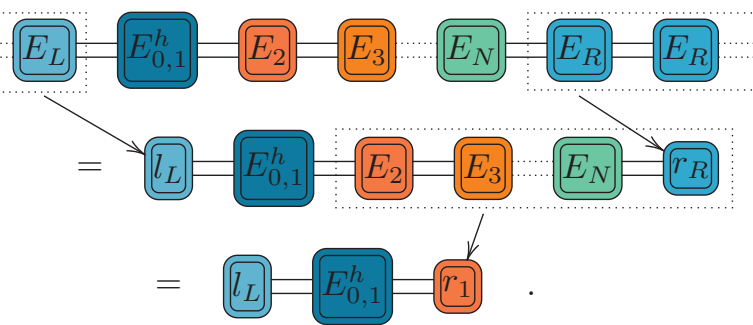

Note that we are free to scale $\left\langle l_{L}|,| r_{R}\right\rangle$ and the tensors $A_{n}$ of the nonuniform region such that $\langle\Psi \mid \Psi\rangle=\left\langle l_{n} \mid r_{n}\right\rangle=1, \forall n$. 
For reasons of efficiency, when constructing numerical algorithms we work in the isomorphic setting where transfer operators are replaced by maps and vectors by matrices using the Choi-Jamiolkowski isomorphism. Here a $D^{2} \times D^{2}$ transfer operator acting on a vector $E_{n}|x\rangle$ becomes $\sum_{s}^{d} A_{n}^{s} x A_{n}^{s \dagger}$ with $x$ a $D \times D$ matrix, so that expectation values can be computed using $O\left(D^{3}\right)$ scalar multiplication operations:

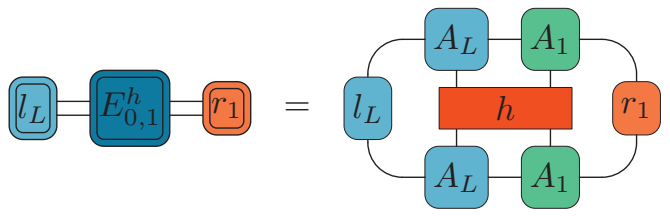

We now determine the dimension of the submanifold $\mathcal{M}_{\text {sMPS }} \subset \mathcal{H}$ of Hilbert space defined by the sMPS variational class. Naively, this is the number of complex entries of the parameter tensors $A_{1 \cdots N}$, which is $N d D^{2}$. However, an sMPS is invariant under gauge transformations

$$
\begin{aligned}
A_{L}^{s} & \rightarrow g_{0} A_{L}^{s} g_{0}^{-1}, \\
A_{1 \leqslant n \leqslant N}^{s} & \rightarrow g_{n-1} A_{n}^{s} g_{n}^{-1}, \\
A_{R}^{s} & \rightarrow g_{N} A_{R}^{s} g_{N}^{-1},
\end{aligned}
$$

with $g_{n} \in M_{D}(\mathbb{C})$. Since $A_{L / R}$ are fixed, we restrict to $g_{0}=$ $g_{N}=\mathbb{I}$ leaving $(N-1) D^{2}$ nonphysical degrees of freedom corresponding to the gauge-transformation matrices $g_{1 \cdots(N-1)}$, as well as a further one corresponding to the norm and phase. The dimension of the sMPS variational manifold is thus $\operatorname{dim}\left(\mathcal{M}_{\mathrm{sMPS}}\right)=[N(d-1)+1] D^{2}-1$. The redundancy in the sMPS representation is familiar from other MPS variational classes $^{9}$ and is less inconvenient than it may appear, since the gauge freedom in the representation of tangent vectors allows for significant simplification of the TDVP flow equations.

To implement the TDVP (see boxout), we must project exact infinitesimal time steps $-i H|\Psi[A]\rangle$ onto the tangent plane $\mathbb{T}_{|\Psi[A]\rangle}$ to $\mathcal{M}_{\text {sMPS }}$ at the point $|\Psi[A]\rangle$. The tangent plane is spanned by tangent vectors

$$
\begin{aligned}
|\Phi[B]\rangle & \\
& =\sum_{n=1}^{N} \sum_{i=1}^{d D^{2}} B_{n, i}\left|\partial_{n, i} \Psi[A]\right\rangle, \\
& =\sum_{n=1}^{N} \cdots \frac{\Psi}{A_{L}-A_{1} \cdots A_{n-1}-B_{n}-A_{n+1} \cdots A_{N}-A_{R} \cdots \cdots},
\end{aligned}
$$

with $\left|\partial_{n, i} \Psi[A]\right\rangle=\partial / \partial A_{n, i}|\Psi[A]\rangle$ and the index $i$ running over all $d D^{2}$ entries of each tensor $A_{n}$ or $B_{n}$. The projection is achieved by finding a $|\Phi[B]\rangle$ that satisfies

$$
|\Phi[B]\rangle=\arg \min _{\left|\Phi\left[B^{\prime}\right]\right\rangle} \| i H|\Psi[A(t)]\rangle+\left|\Phi\left[B^{\prime}\right]\right\rangle \|^{2} .
$$

Expanding the right-hand side leaves terms $\langle\Phi[\bar{B}] \mid \Phi[B]\rangle$ and $\langle\Phi[\bar{B}]|H| \Psi[A]\rangle+$ H.c., where the remaining $H^{2}$ term is a constant that can be ignored. The metric term $\langle\Phi[\bar{B}] \mid \Phi[B]\rangle$ is at first glance very complicated, since it couples the tensors
$B_{n}$ for different lattice sites in terms such as
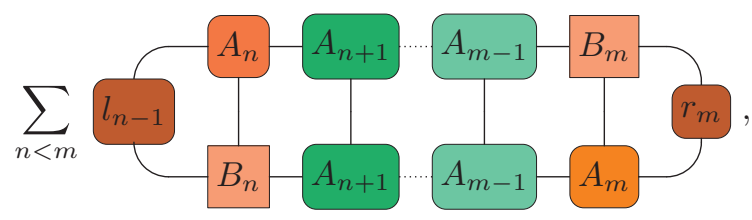

precluding a splitting of the problem into $N$ separate parts (one for each $B_{n}$ ). Fortunately, these site-mixing terms can be eliminated by fixing the gauge freedom in the tangent vector representation. If we impose the left gauge-fixing conditions (GFCs)

$$
\left\langle l_{n-1}\right| E_{A_{n}}^{B_{n}}=0=\sum_{s}^{d} A_{n}^{s \dagger} l_{n-1} B_{n}^{s}
$$

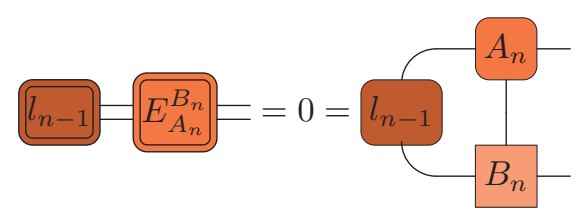

for sites $1 \leqslant n<n_{c}$ and the right gauge-fixing conditions

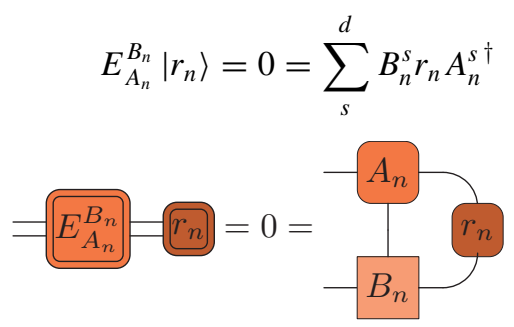

for sites $n_{c}<n \leqslant N$, we eliminate all site-mixing terms like (5) such that $\langle\Phi[\bar{B}] \mid \Phi[B]\rangle=\sum_{n=1}^{N}\left\langle l_{n-1}\left|E_{B_{n}}^{B_{n}}\right| r_{n}\right\rangle$. Note that, for some site $n_{c}$ in the nonuniform region, the tangent vector parameters $B_{n_{c}}$ are not constrained. For reasons of symmetry, we choose $n_{c}$ to be in the middle so that $2\left(n_{c}-1\right)+1=N$ with odd $N$.

To see that the conditions (6) and (7) fix exactly the gauge degrees of freedom, we consider the one-parameter gauge transformation $g_{n}(\eta)=\mathbb{I}+\eta x_{n} \quad \forall n \in[0 \cdots N]$ with $x_{0}=x_{N}=0$. Writing the transformed state as $\left|\Psi\left[A^{\prime}(\eta)\right]\right\rangle$, the infinitesimal transformation has the form (3) of a tangent vector

$$
\left.\frac{d}{d \eta}\left|\Psi\left[A^{\prime}(\eta)\right]\right\rangle\right|_{\eta=0}=|\Phi[\mathcal{N}[x]]\rangle=0,
$$

with $\mathcal{N}_{n}^{s}[x]=x_{n-1} A_{n}^{s}-A_{n}^{s} x_{n}$. Tangent vector parameters of this form thus capture exactly the gauge freedom so that an arbitrary tangent vector fulfills $|\Phi[B]\rangle=|\Phi[B+\mathcal{N}[x]]\rangle$. Using this freedom, we can always transform arbitrary $B_{n}^{\prime}$ as $B_{n}=B_{n}^{\prime}+\mathcal{N}_{n}[x]$ so that $B_{n}$ satisfies the gauge-fixing conditions (6) and (7). To see this, we insert $B_{n}^{\prime}+\mathcal{N}_{n}[x]$ into (7) to obtain

$$
x_{n-1} r_{n-1}=\sum_{s}^{d}\left(A_{n}^{s} x_{n}-B_{n}^{\prime s}\right) r_{n} A_{n}^{s \dagger} \quad \forall n>n_{c},
$$

which we can solve to fully determine $x_{n-1}$ given that $r_{n-1}$ has full rank and that $x_{n}$ is known. Starting at $n=N$ with $x_{N}=0$, 
this fixes all $x_{n}$ down to $n=n_{c}$. We can perform the same trick with (6) to get

$$
l_{n} x_{n}=\sum_{s}^{d} A_{n}^{s \dagger} l_{n-1}\left(x_{n-1} A_{n}^{s}+B_{n}^{\prime s}\right) \quad \forall n<n_{c},
$$

which determines the remaining $x_{n}$ (up to $n=n_{c}-1$ ) given that $x_{0}=0$ and that $l_{n}$ has full rank.

We can construct $B_{n}$ such that they automatically fulfill the GFCs (6) and (7). For $n_{c}<n \leqslant N$ we define the $(d-1) D \times$ $d D$ matrix $V_{n}^{\dagger}$ to contain an orthonormal basis for the null space of $\left[R_{n}\right]_{(\alpha, s) ; \beta}=\left[r_{n}^{1 / 2} A_{n}^{s \dagger}\right]_{\alpha, \beta}$ and set

$$
B_{n}^{s}\left(x_{n}\right)=l_{n-1}^{-1 / 2} x_{n} V_{n}^{s} r_{n}^{-1 / 2} \quad \forall n \in\left[n_{c}+1, N\right],
$$

with parameters $x_{n}$. For $1 \leqslant n<n_{c}$ we define the $d D \times(d-$ 1) $D$ matrix $W_{n}$ to contain an orthonormal basis for the null space of $\left[L_{n}\right]_{\alpha ;(s, \beta)}=\left[A_{n}^{s \dagger} l_{n-1}^{1 / 2}\right]_{\alpha, \beta}$ and set

$$
B_{n}^{s}\left(x_{n}\right)=l_{n-1}^{-1 / 2} W_{n}^{s} x_{n} r_{n}^{-1 / 2} \quad \forall n \in\left[1, n_{c}-1\right] .
$$

It is easy to check by insertion that (9) and (8), respectively, satisfy the GFCs (6) and (7). Note again that $B_{n_{c}}$ remains unconstrained. Using the parametrizations, we obtain

$$
\langle\Phi[\bar{B}] \mid \Phi[B]\rangle=\sum_{n \neq n_{c}} \operatorname{tr}\left[x_{n}^{\dagger} x_{n}\right]+\left\langle l_{n_{c}-1}\left|E_{B_{n_{c}}}^{B_{n_{c}}}\right| r_{n_{c}}\right\rangle .
$$

Having fixed the gauge, one nonphysical degree of freedom remains, since $\langle\Psi[\bar{A}] \mid \Phi[B]\rangle=\left\langle l_{n_{c}-1}\left|E_{A_{n_{c}}}^{B_{n_{c}}}\right| r_{n_{c}}\right\rangle \neq 0$, implying that the tangent plane contains infinitesimal changes to the norm and phase. We must thus explicitly eliminate norm and phase changes when implementing the TDVP, which can be done by replacing $H$ with $\tilde{H} \equiv H-\langle\Psi|H| \Psi\rangle$ in the TDVP flow equations, effectively projecting out the corresponding component of $H|\Psi\rangle .^{9}$

With gauge fixing, $\langle\Phi[\bar{B}]|\tilde{H}| \Psi[A]\rangle$ simplifies, but still contains terms mixing $B_{n}$ and $\tilde{h}_{m, m+1} \equiv h_{m, m+1}-\left\langle h_{m, m+1}\right\rangle$ for $m, m+1 \neq n$. Each $B_{n}$ term contains a sum over $\tilde{h}_{m, m+1}$ extending into the left $\left(n<n_{c}\right)$ or right $\left(n>n_{c}\right)$ bulk or into both $\left(n=n_{c}\right)$. This is understood by defining the right and left effective Hamiltonians

$$
\begin{aligned}
\left|K_{n+1}\right\rangle & =\sum_{m=n+1}^{\infty}\left(\prod_{k=n+1}^{m-1} E_{k}\right) E_{m}^{\tilde{h}}\left|r_{m+1}\right\rangle, \\
\left\langle J_{n-1}\right| & =\sum_{m=-\infty}^{n-2}\left\langle l_{m-1}\right| E_{m}^{\tilde{h}}\left(\prod_{k=m+2}^{n-1} E_{k}\right),
\end{aligned}
$$

which also obey

$$
\begin{aligned}
\left|K_{n}\right\rangle & =E_{n}\left|K_{n+1}\right\rangle+E_{n}^{\tilde{h}}\left|r_{n+1}\right\rangle, \\
\left\langle J_{n}\right| & =\left\langle J_{n-1}\right| E_{n}+\left\langle l_{n-2}\right| E_{n-1}^{\tilde{h}},
\end{aligned}
$$

where $\quad E_{n}^{\tilde{h}}=\sum_{s, t=1}^{d} C_{n}^{s, t} \otimes \overline{A_{n}^{s} A_{n+1}^{t}} \quad$ and $\quad C_{n}^{s, t}=$ $\sum_{u, v=1}^{d}\left\langle s, t\left|\tilde{h}_{n, n+1}\right| u, v\right\rangle A_{n}^{u} A_{n+1}^{v}$. For example, the terms containing $B_{n}$ with $n>n_{c}$ are

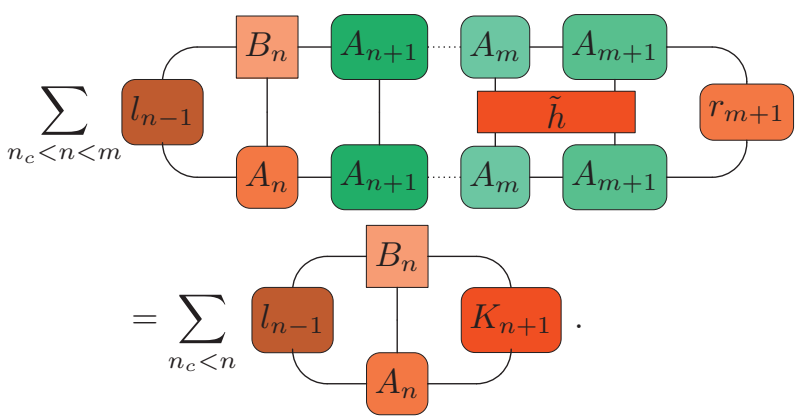

The sums over the uniform bulk $\left|K_{R}\right\rangle \equiv\left|K_{N+1}\right\rangle=$ $\sum_{n=0}^{\infty}\left(E_{R}\right)^{n} E_{R}^{\tilde{h}}\left|r_{R}\right\rangle$ and $\left\langle J_{L}\right| \equiv\left\langle J_{0}\right|=\sum_{n=0}^{\infty}\left\langle l_{L}\right| E_{L}^{\tilde{h}}\left(E_{L}\right)^{n}$ can be computed by exploiting the assumption that $E_{L / R}$ have a unique largest (in magnitude) eigenvalue equal to 1 , which allows us to rewrite the sum as a pseudoinverse. For the righthand bulk this gives $\left|K_{R}\right\rangle=\left(\mathbb{I}-E_{R}\right)^{\mathrm{P}} E_{R}^{\tilde{h}}\left|r_{R}\right\rangle$ or, equivalently, $\left[\mathbb{I}-\left(E_{R}-\left|r_{R}\right\rangle\left\langle l_{R}\right|\right)\right]\left|K_{R}\right\rangle=E_{R}^{\tilde{h}}\left|r_{R}\right\rangle$, which can then be solved for $K_{R}$ in the matrix representation using $O\left(D^{3}\right)$ operations per iteration. $\left\langle J_{L}\right|$ can be computed analogously. Note that the energy difference due to the nonuniformity is $\Delta E=$ $\left\langle J_{L} \mid r_{0}\right\rangle+\left\langle l_{N} \mid K_{R}\right\rangle+\sum_{n=0}^{N}\left\langle h_{n, n+1}\right\rangle-(N+1)\langle h\rangle_{\text {uni }}$, where $\langle h\rangle_{\text {uni }}$ is the energy per site of the uniform bulk state.

We now have the ingredients needed to compute the Hamiltonian term efficiently as

$$
\begin{aligned}
& \langle\Phi[\bar{B}]|\tilde{H}| \Psi[A]\rangle \\
& \quad=\sum_{n \neq n_{c}} \operatorname{tr}\left[x_{n}^{\dagger} F_{n}\right]+\sum_{s=1}^{d} \operatorname{tr}\left[l_{n_{c}-1} G_{n_{c}}^{s} r_{n_{c}} B_{n_{c}}^{s \dagger}\right],
\end{aligned}
$$

with

$$
\begin{aligned}
F_{n>n_{c}} \equiv & \sum_{s, t}^{d} l_{n-1}^{1 / 2} C_{n}^{s, t} r_{n+1} A_{n+1}^{t}{ }^{\dagger} r_{n}^{-1 / 2} V_{n}^{s \dagger} \\
& +\sum_{s, t}^{d} l_{n-1}^{-1 / 2} A_{n-1}^{t}{ }^{\dagger} l_{n-2} C_{n}^{t, s} r_{n}^{1 / 2} V_{n}^{s \dagger} \\
& +\sum_{s}^{d} l_{n-1}^{1 / 2} A_{n}^{s} K_{n+1} r_{n}^{-1 / 2} V_{n}^{s \dagger}, \\
F_{n<n_{c}} \equiv & \sum_{s, t}^{d} W_{n}^{s \dagger} l_{n-1}^{1 / 2} C_{n}^{s, t} r_{n+1} A_{n+1}^{t}{ }^{\dagger} r_{n}^{-1 / 2} \\
& +\sum_{s, t}^{d} W_{n}^{s \dagger} l_{n-1}^{-1 / 2} A_{n-1}^{t}{ }^{\dagger} l_{n-2} C_{n-1}^{t, s} r_{n}^{1 / 2} \\
& +\sum_{s}^{d} W_{n}^{s \dagger} l_{n-1}^{-1 / 2} J_{n-1} A_{n}^{s} r_{n}^{1 / 2}, \\
G_{n_{c}}^{s} \equiv & A_{n_{c}}^{s} K_{n_{c}+1} r_{n_{c}}^{-1}+l_{n_{c}-1}^{-1} J_{n_{c}-1} A_{n_{c}}^{s} \\
& +\sum_{t}^{d}\left[C_{n_{c}}^{s, t} r_{n_{c}+1} A_{n_{c}+1}^{t}{ }^{\dagger} r_{n_{c}}^{-1}\right. \\
& \left.+l_{n_{c}-1}^{-1} A_{n_{c}-1}^{t}{ }^{\dagger} l_{n_{c}-2} C_{n_{c}-1}^{t, s}\right],
\end{aligned}
$$


where $n \in[1, N]$ and $J_{n}$ is the conjugate matrix representation of $\left\langle J_{n}\right|$ so that, for some vector $|y\rangle,\left\langle J_{n} \mid y\right\rangle=\operatorname{tr}\left[J_{n} y\right]$.

Having fixed the gauge, inserting (10) and (11) into the TDVP minimization problem (4) and minimizing over the parameters $x_{n \neq n_{c}}$ and $B_{n_{c}}$ gives us $N-1+d$ independent matrix equations,

$$
B_{n_{c}}^{s}=-\mathrm{i} G_{n_{c}}^{s} \quad(s \in[1, d]) \quad \text { and } \quad x_{n}=-i F_{n} \quad\left(n \neq n_{c}\right),
$$

representing the optimal time evolution for the variational parameters

$$
\dot{A}_{n_{c}}^{s}=-\mathrm{i} G_{n_{c}}^{s}(A) \quad \text { and } \quad \dot{A}_{n \neq n_{c}}^{s}=B_{n}^{s}\left[-i F_{n}(A)\right],
$$

where we use the appropriate parametrization (8) or (9) for $B_{n}$ depending on the value of $n$. With gauge fixing, the independent terms to be minimized in (4), one for each $B_{n}$, can be summarized diagrammatically as
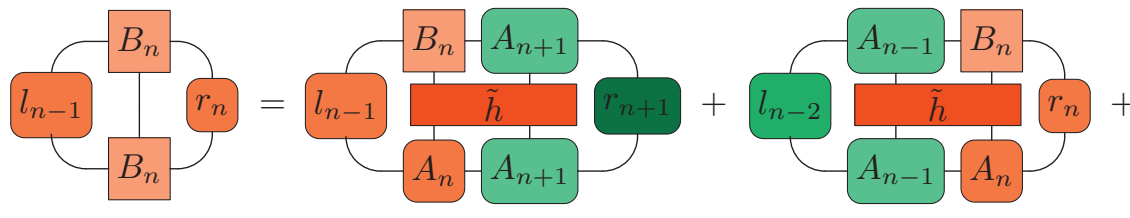
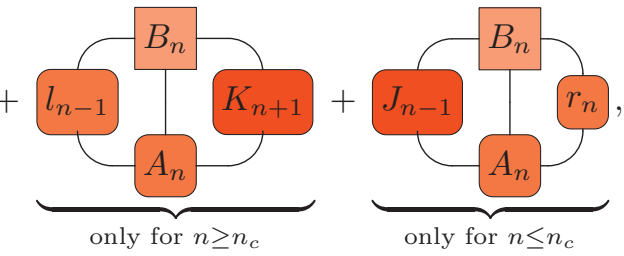

where the equations for $x_{n}$ are obtained again by replacing $B_{n}$ with (8) or (9) for $n \neq n_{c}$ as appropriate. The flow equations (12) can be integrated numerically, for example with the following simple algorithm implementing the Euler method.

(1) Calculate $F_{n}, G_{n_{c}}^{s}$.

(2) Take a step by setting $A_{n}(t+d t)=A_{n}(t)+d t B_{n}$.

(3) Restore a canonical form using a gauge transformation (2) and normalize the state by rescaling $A_{n_{c}}$.

(4) Compute desired quantities, such as the energy expectation value, and adjust the step size $d t$ as required.

(5) If needed, expand the nonuniform region to the left and/or right.

Normalization is necessary because the norm is only preserved to first order in $d t$. Maintaining a canonical form (for example, see Appendix A) can simplify some parts of the TDVP calculations and improve the conditioning of the matrices involved. The last step allows for a small initial nonuniform region, which can be grown if the dynamics warrant changing the state significantly outside of it. This is done by "absorbing" sites from the uniform region(s) into the nonuniform region, copying the $A_{L}$ and $A_{R}$ matrices as needed.

Whether it is necessary to grow the nonuniform region can be heuristically determined by observing the per-site contributions $\eta_{n}=\sqrt{\left\langle l_{n-1}\left|E_{B_{n}}^{B_{n}}\right| r_{n}\right\rangle}$ to the norm $\eta=\sum_{n} \eta_{n}$ of the TDVP tangent vector $|\Phi[B]\rangle$. If $\eta_{1}$ and $\eta_{N}$ become significantly larger than the norm $\eta_{\text {uni }}$ of the uniform MPS TDVP tangent vector of the bulk state then the nonuniform region should be expanded until this is no longer the case.

Note also that the above algorithm is not well suited to simulating real-time dynamics because errors due to the simple integration method used are cumulative. Instead, more sophisticated integrators such as the commonly used fourth-order explicit Runge-Kutta method (see Appendix B) are preferable. The Euler method is, however, still useful for finding ground states because imaginary time evolution is "self-correcting"-it will always take you towards the ground state, given that the starting point is not orthogonal to it.
To test our algorithm, we use the antiferromagnetic spin-1 Heisenberg model $h_{n, n+1}^{\mathrm{uni}}=h_{n, n+1}^{\mathrm{AFH}}$, with

$$
h_{n, n+1}^{\mathrm{AFH}}=\sum_{i=x, y, z} S_{n}^{i} S_{n+1}^{i} .
$$

The uniform ground state respects the SU(2) symmetry of the Hamiltonian. Having found a uniform MPS approximation for the ground state, we use imaginary time evolution to find the ground state of a nonuniform model where one of the coupling terms has its sign flipped via the addition of $h_{0,1}^{\text {loc }}=-2 h_{0,1}^{\mathrm{AFH}}$, with all other $h_{n \neq 0, n+1}^{\text {loc }}=0$, thus creating a ferromagnetic impurity. Impurities have been studied in this model before, ${ }^{15}$ however, to the best of our knowledge the case of a ferromagnetic bond has not yet been investigated. It appears to lead to localized SU(2) symmetry breaking, as can be seen in the relative distribution of the spin expectation values at each site, which we plot in Fig. 1. This is expected, since the ground states of the uniform ferromagnetic model also break the symmetry. In this case, $-\sum_{i} S_{0}^{i} S_{1}^{i}$ acts in the

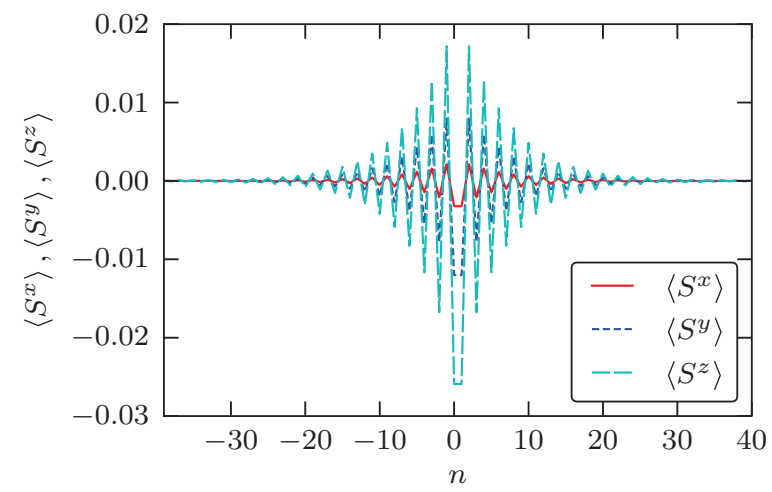

FIG. 1. (Color online) Per-site spin expectation values of the approximate ground state of the spin-1 antiferromagnetic Heisenberg model with a ferromagnetic impurity. The state was converged up to $\eta \approx 3 \times 10^{-8}$ with $D=64$ and a nonuniform region $[-100,100]$. The initial uniform ground state used for the left and right bulk parts was converged to $\eta \approx 10^{-12}$. 


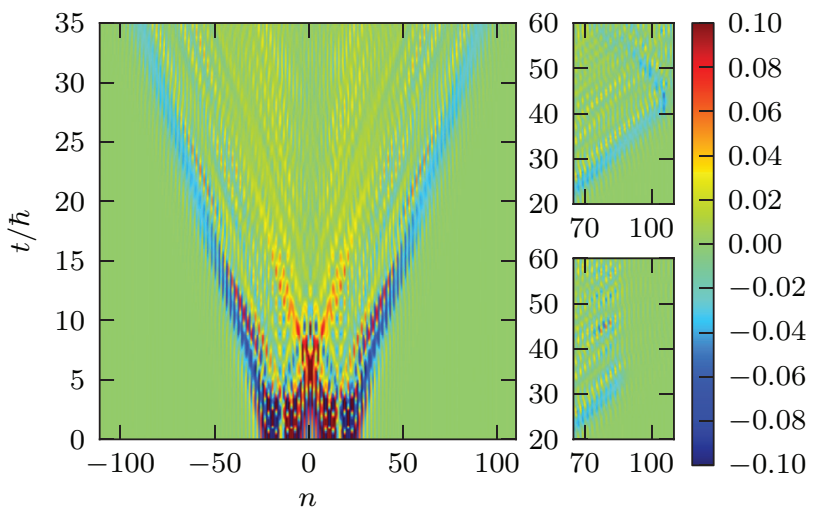

FIG. 2. (Color online) Simulated real time evolution (using a fixed nonuniform region $[-100,100]$ with $D=64$ and $d t=0.04$ ) of the spin-1 antiferromagnetic Heisenberg model with two localized entangled excitations generated by applying $S_{m-j}^{-} S_{m+j}^{+}$at $m= \pm 15$ with $j= \pm 5$. The plots show the expectation value of $S_{n}^{z}$ with the top-right plot showing the excitation bouncing at the right boundary. For the bottom-right plot, we used a Gaussian optical potential to suppress this reflection, albeit imperfectly, with $\epsilon_{n}=$ $e^{-(n+90)^{2} / 18}+e^{-(n-90)^{2} / 18}$. The uniform ground state was converged up to a state tolerance $\eta=10^{-8}$. For the time integration we used a fourth order explicit Runge-Kutta algorithm.

Hamiltonian to approximately project the pair of sites 0 and 1 onto the spin 2 subspace, whose states are not invariant under SU(2).

As a test of real-time evolution, we again use $h^{\text {uni }}=h_{n, n+1}^{\mathrm{AFH}}$ from (13), but without any local perturbations $\left(h_{n, n+1}^{\text {loc }}=0\right.$, $\forall n)$. We begin with a uniform ground state approximation and introduce local excitations by applying the (nonunitary) operator $S_{m-j}^{-} S_{m+j}^{+}$with $S_{n}^{ \pm}=S^{x} \pm \mathrm{i} S^{y}$, which generates an entangled excitation, to two separated pairs of sites at $m= \pm k$ inside a nonuniform region. By calculating the expectation value of an observable such as $S^{z}$ for a set of sites (possibly extending into the left and right bulk regions) after each step, the time evolution of the system can be visualized, for example by plotting the site spin expectation values as in Fig. 2 or the half-chain entropy for splittings at each site as in Fig. 3. For the latter we use dynamic expansion of the nonuniform region to maximize numerical efficiency. Note that the entropy for a splitting after site 0 appears to tend to an asymptotic value of approximately 3.5. This suggests that a hybrid method, whereby uniform matrices are reintroduced between the two excitations as they become separated, could be used to study the asymptotics of entangled excitations for large times.

To mitigate nonphysical reflections that can occur when a traveling excitation meets a boundary with the uniform region, "optical potential" terms $h_{n, n+1}^{\text {loc }}=-\mathrm{i} \epsilon_{n} h_{n, n+1}^{\text {uni }}$ can be locally turned on near to the boundaries. This effectively carries out imaginary time evolution on a subsystem defined by the envelope function $\epsilon(n)$, where the magnitude of $\epsilon(n)$ determines the rate of "cooling" at each site. If $\epsilon(n)$ is a step function that turns on imaginary time evolution at a constant rate in a small part of the lattice, that part should (in the absence of simultaneous real time evolution) converge to the ground state of a finite chain with open boundary conditions. Since we are working with gapped systems, the ground state of a

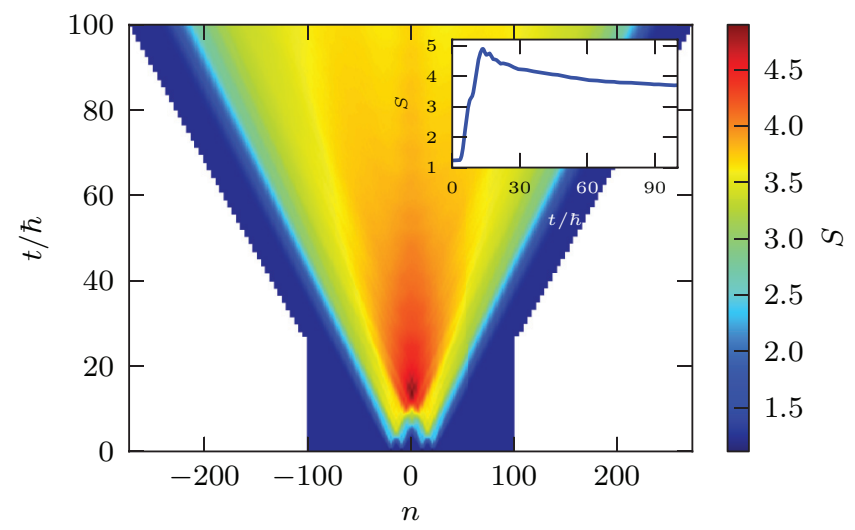

FIG. 3. (Color online) The time evolution of the block entropy $S$ of one half of the lattice, split at each site $n$, for the same simulation as in Fig. 2, except that dynamic expansion of the nonuniform region is used, as indicated by the "staircase" pattern-the bulk parts are shown in white. The inset shows a cross section at site 0 .

smaller part should be the same as that of the uniform infinite system up to boundary effects. We find that choosing $\epsilon(n)$ to be superposition of two Gaussians, each localized near an edge of the nonuniform region, avoids significant boundary effects during evolution of the Heisenberg model (13) while successfully attenuating boundary reflections, as shown in Fig. 2. Note that the entanglement present in the excitations produced for this particular model mean that the boundary absorption affects the evolution in the central region as well as at the boundaries themselves. Further tuning of $\epsilon_{n}$ may help to more effectively dissipate the excitations heading out of the nonuniform region.

As a final test of our approach we simulate the scattering of localized excitations in $\phi^{4}$ theory on a one-dimensional lattice. The Hamiltonian is

$$
H^{\phi^{4}}=\sum_{n}\left(\frac{1}{2} \pi_{n}^{2}+\frac{\tilde{\mu}_{0}^{2}}{2} \phi_{n}^{2}+\frac{\tilde{\lambda}}{4 !} \phi_{n}^{4}+\frac{1}{2}\left(\phi_{n}-\phi_{n+1}\right)^{2}\right),
$$

where $\left[\phi_{n}(t), \pi_{m}(t)\right]=\mathrm{i} \delta_{n m}$. The bare mass $\tilde{\mu}_{0}$ and coupling $\tilde{\lambda}$ are dimensionless lattice parameters related to parameters with dimension [mass] $]^{2}$ by $\tilde{\mu}_{0}^{2}=a^{2} \mu_{0}^{2}, \tilde{\lambda}=a^{2} \lambda$, where $a$ is the lattice spacing. We fix $a$ for each set of parameters using the ground state correlation length in lattice sites $\tilde{\xi}$, which is directly obtainable ${ }^{16}$ from the largest two eigenvalues of the uniform MPS transfer operator $E$. Due to renormalization, $\tilde{\mu}_{0}$ is not equal to the particle mass and in fact diverges in the continuum limit. So that our parameters are well defined in the limit, we separate out the divergent contribution $\delta \tilde{\mu}^{2}$ to obtain the renormalized mass-squared parameter $\tilde{\mu}_{R}^{2}=\tilde{\mu}_{0}^{2}-$ $\delta \tilde{\mu}^{2}$. For certain values of $\tilde{\mu}_{R}^{2}, \tilde{\lambda}$ the ground state spontaneously breaks the global $\mathbb{Z}_{2}$ symmetry $\phi_{n}=-\phi_{n}$ of (14) such that $\left\langle\phi_{n}\right\rangle= \pm \phi_{0}$. In Fig. 4 we examine excitations of $\phi^{4}$ theory within a nonuniform region by applying the field operator to the ground state and simulating time evolution. We do this for a sequence of parameters, approaching a continuum limit. More details about the application of MPSs to real scalar $\phi^{4}$ theory and its critical behavior are available elsewhere. ${ }^{17,18}$ 


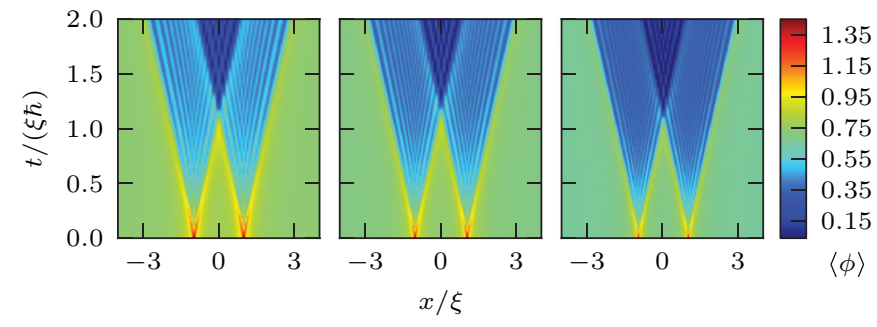

FIG. 4. (Color online) Simulation $(D=24, d=16, d t=0.02)$ of particle scattering in $(1+1)$-dimensional lattice $\phi^{4}$ real scalar field theory approaching a continuum limit (from left to right), as determined by the ground-state correlation length in lattice sites $\tilde{\xi}$. We created two excitations by applying the field operator $\phi_{n}$ at two different sites $n= \pm[\tilde{\xi}]$ to an approximate ground state in the symmetry-broken phase. The coupling is $\tilde{\lambda}=0.2$ for all three plots and the parameter ratio $\tilde{\lambda} / \tilde{\mu}_{R}^{2}$ varies from left to right as $\tilde{\lambda} / \tilde{\mu}_{R}^{2}=85,80,75$. Distance $x$ and time $t$ are scaled with the correlation length $\xi=a \tilde{\xi}$, where $a$ is the lattice spacing. The field expectation value $\langle\phi\rangle$ is left unscaled, although a more comprehensive treatment would scale it with the field strength renormalization factor, which can also be computed from the uniform MPS approximate ground state.

In this paper we have introduced an efficient means of simulating the dynamics of localized nonuniformities on spin chains in the thermodynamic limit using the time-dependent variational principle (TDVP) and a special class of matrix product states (MPSs). As with the existing algorithms implementing the TDVP for MPSs in other settings, ${ }^{9}$ this algorithm approximates exact time evolution optimally given the restrictions of the variational class. Our (open source) implementation evoMPS ${ }^{19}$ is available as Python (http://www.python.org) source code, including example simulation scripts.

During completion of this work we learned of other independent results ${ }^{20-22}$ that use time-evolving block decimation to approximate the time evolution of a nonuniform window on an otherwise translation-invariant chain. Our approach differs in that we define a variational class and apply the TDVP to obtain equations for locally optimal approximate time evolution. We are then able to apply standard numerical integration techniques. The idea of not only growing the nonuniform region, but also of ignoring the evolution of uninteresting parts of the nonuniformity for reasons of efficiency, say to follow a wave front, ${ }^{21,22}$ can also be implemented in our scheme by restricting the variational parameters to a smaller part of the nonuniform region and leaving the rest constant (up to gauge transformations). As mentioned above, another approach to studying entangled excitations may be to detect when the central region between two separating wave fronts becomes translation invariant over a sufficiently large region, taking this state as a new bulk state for one side of the system and restricting the nonuniform region to a single wave front.

Helpful discussions with Florian Richter, Fabian Transchel, and Fabian Furrer are gratefully acknowledged. This work was supported by the ERC Grants QFTCMPS, QUERG, and QUEVADIS, the FWF SFB Grants FoQuS and ViCoM, and the cluster of excellence EXC 201 Quantum Engineering and Space-Time Research.

\section{APPENDIX A: CANONICAL FORM}

A canonical form that fits to the gauge-fixing conditions (GFCs) (6) and (7) is given by

$$
\begin{aligned}
l_{n} & =\mathbb{I} \quad \forall n<n_{c}, \\
l_{n} & =\operatorname{diag}\left(\lambda_{n, 1}^{2} \cdots \lambda_{n, D}^{2}\right) \quad \forall n \in\left[n_{c}, N\right], \\
r_{n} & =\mathbb{I} \quad \forall n \geqslant n_{c}, \\
r_{n} & =\operatorname{diag}\left(\lambda_{n, 1}^{2} \cdots \lambda_{n, D}^{2}\right) \quad \forall n \in\left[0, n_{c}-1\right],
\end{aligned}
$$

where $\lambda_{n, i}$ for $1 \leqslant i \leqslant D$ are the Schmidt coefficients for the decomposition of the chain into two infinite halves by cutting between sites $n$ and $n+1$. It corresponds to the GFCs in the sense that changing the parameters as $A_{n} \rightarrow A_{n}+\epsilon B_{n}$ with $B_{n}$ satisfying the GFCs does not alter $l_{n<n_{c}}$ or $r_{n \geqslant n_{c}}$, which are constants in the above canonical form, to first order in $\epsilon$. In practice, this means that the canonical form is approximately maintained when making finite steps in the TDVP algorithm.

The above canonical form can be reached via a gaugetransformation $g_{0 \ldots N}$, where $g_{0}$ and $g_{N}$ are nontrivial [see (2)], such that the uniform bulk parameters $A_{L / R}$ are also transformed. Since the overall state and also the left and right uniform bulk states are unaffected by these transformations, performing them does not affect evolution under the TDVP equations.

\section{APPENDIX B: RUNGE-KUTTA INTEGRATION}

For real-time evolution, numerical integration using the Euler method is inefficient since small step sizes $d t$ are required to keep the $O\left(d t^{2}\right)$ integration errors made with each finite step small. A well known integration method with more favorable error scaling is the fourth order Runge-Kutta method (RK4), ${ }^{23}$ which makes per-step errors $O\left(d t^{5}\right)$ at the cost of three extra evaluations of the derivative. It builds a final step by making three smaller steps and weighting the derivatives obtained at the visited points. Given a differential equation $\dot{\boldsymbol{a}}=$ $f(t, \boldsymbol{a})$, the RK4 method estimates $\boldsymbol{a}(t+d t) \approx \boldsymbol{a}(t)+d t \boldsymbol{b}_{\mathrm{RK} 4}$ with $\boldsymbol{b}_{\mathrm{RK} 4} \equiv \frac{1}{6}\left(\boldsymbol{b}_{1}+2 \boldsymbol{b}_{2}+2 \boldsymbol{b}_{3}+\boldsymbol{b}_{4}\right)$ and

$$
\begin{aligned}
& \boldsymbol{b}_{1}=f[t, \boldsymbol{a}(t)], \\
& \boldsymbol{b}_{2}=f\left[t+\frac{d t}{2}, \boldsymbol{a}(t)+\frac{d t}{2} \boldsymbol{b}_{1}\right], \\
& \boldsymbol{b}_{3}=f\left[t+\frac{d t}{2}, \boldsymbol{a}(t)+\frac{d t}{2} \boldsymbol{b}_{2}\right], \\
& \boldsymbol{b}_{4}=f\left[t+d t, \boldsymbol{a}(t)+d t \boldsymbol{b}_{3}\right] .
\end{aligned}
$$

The sMPS TDVP flow equations derived in the main part of this work provide the derivative function for the $n$th site $B_{n}=f_{n}(t,[A])$, allowing us to implement the RK4 integrator without any additional tools. It is worth noting that $B_{\mathrm{RK} 4}$, obtained by adding the tangent vector parameters from the various substeps, is not gauge fixing. This is because each individual $B_{n, i}$, although it is gauge fixing for the substep point $A^{\prime}$ at which it was obtained, is not generally gauge fixing when applied at the original point $A$. Additionally, each substep changes the gauge choice slightly, since gauge fixing only holds to first order in the step size. On the other hand, since the gauge-fixing flow equations do preserve the gauge choice 
when integrated exactly, gauge fixing should improve with the accuracy of the numerical integration. We should thus expect the RK4 method to maintain the gauge choice up to errors of $O\left(d t^{5}\right)$ with each step. This is far better than the Euler method, which incurs $O\left(d t^{2}\right)$ errors.

The error can be quantified by the change in the energy expectation value, which is conserved under exact time evolution. We confirm the benefits of our RK4 implementation by comparing it to the Euler method for the Heisenberg model example described in the main text, which we simulate on a finite chain with open boundary conditions in order to avoid errors due to the interface with the bulk. To compare the efficiency of the two methods, we set the step sizes such that the computation time per unit simulated time is roughly the same and examine the overall change in the energy expectation value after a period of simulated time $T$. Since a single RK4 step requires roughly four times as much computation as an Euler step, we choose $d t_{\mathrm{RK} 4}=4 d t_{\text {Euler. For }} d t_{\mathrm{RK} 4}=0.01$, the energy errors after a time $T=10 \hbar s$ are $\epsilon_{\text {Euler }}=-1.01 \times 10^{-3}$ and $\epsilon_{\mathrm{RK} 4}=-9 \times 10^{-6}$, showing a significant advantage for the RK4 method for the same computation time. The vast majority of the RK4 error comes from the first four steps, whereas the Euler errors are uniformly distributed in time. Excluding these steps from the RK4 error estimate results in $\epsilon_{\mathrm{RK} 4}^{\prime}=-3 \times 10^{-9}$. Both $\epsilon_{\mathrm{RK} 4}^{\prime}$ and $\epsilon_{\text {Euler }}$ are in line with the theoretical global error estimates of $O\left(d t_{\mathrm{RK} 4}^{4}\right)$ and $O\left(d t_{\text {Euler }}\right)$, respectively. The comparatively large errors made by the RK4 method during the first few steps are caused by the presence of particularly small Schmidt coefficients, indicating that the bond dimension is higher than necessary. Small Schmidt coefficients lead to instability because the squares of the Schmidt coefficients appear in the $l$ and $r$ matrices, which are inverted in the TDVP algorithm, amplifying errors on small values greatly. To mitigate this, the bond dimension can be reduced dynamically (and increased later if necessary), cutting off Schmidt coefficients that are close to zero. Alternatively, an integrator that is robust under low-rank conditions could be used. $^{24}$ *ashley.milsted@itp.uni-hannover.de

${ }^{1}$ J. Eisert, M. Cramer, and M. B. Plenio, Rev. Mod. Phys. 82, 277 (2010).

${ }^{2}$ M. B. Hastings, J. Stat. Mech. (2007) P08024.

${ }^{3}$ T. J. Osborne, Phys. Rev. Lett. 97, 157202 (2006).

${ }^{4}$ M. Fannes, B. Nachtergaele, and R. F. Werner, Commun. Math. Phys. (1965-1997) 144, 443 (1992); F. Verstraete, V. Murg, and J. Cirac, Adv. Phys. 57, 143 (2008); J. I. Cirac and F. Verstraete, J. Phys. A 42, 504004 (2009).

${ }^{5}$ S. R. White, Phys. Rev. Lett. 69, 2863 (1992); U. Schollwöck, Rev. Mod. Phys. 77, 259 (2005).

${ }^{6}$ S. Rommer and S. Ostlund, Phys. Rev. B 55, 2164 (1997);

U. Schollwöck, Ann. Phys. 326, 96 (2011).

${ }^{7}$ G. Vidal, Phys. Rev. Lett. 93, 040502 (2004).

${ }^{8}$ F. Verstraete and J. I. Cirac, arXiv:cond-mat/0407066.

${ }^{9}$ J. Haegeman, J. I. Cirac, T. J. Osborne, I. Pižorn, H. Verschelde, and F. Verstraete, Phys. Rev. Lett. 107, 070601 (2011).

${ }^{10}$ V. Nebendahl and W. Dür, Phys. Rev. B 87, 075413 (2013).

${ }^{11}$ M. B. Hastings, J. Math. Phys. 50, 095207 (2009).

${ }^{12}$ M. C. Bañuls, M. B. Hastings, F. Verstraete, and J. I. Cirac, Phys. Rev. Lett. 102, 240603 (2009).

${ }^{13}$ J. A. Kjäll, F. Pollmann, and J. E. Moore, Phys. Rev. B 83, 020407 (2011).
${ }^{14}$ J. Haegeman, M. Mariën, T. J. Osborne, and F. Verstraete, arXiv:1210.7710.

${ }^{15}$ M. Kaburagi, I. Harada, and T. Tonegawa, J. Phys. Soc. Jpn. 62, 1848 (1993); M. Kaburagi and T. Tonegawa, ibid. 63, 420 (1994); E. S. Sørensen and I. Affleck, Phys. Rev. B 51, 16115 (1995); W. Wang, S. Qin, Z. Y. Lu, L. Yu, and Z. Su, ibid. 53, 40 (1996); X. Wang and S. Mallwitz, ibid. 53, R492 (1996).

${ }^{16}$ J. Haegeman, T. J. Osborne, and F. Verstraete, Phys. Rev. B 88, 075133 (2013)

${ }^{17}$ T. Sugihara, J. High Energy Phys. 05 (2004) 007.

${ }^{18}$ A. Milsted, J. Haegeman, and T. J. Osborne, arXiv:1302.5582.

${ }^{19}$ A. Milsted, GitHub (EVOMPS computer source code), http:// amilsted.github.io/evoMPS/.

${ }^{20}$ H. N. Phien, G. Vidal, and I. P. McCulloch, Phys. Rev. B 86, 245107 (2012).

${ }^{21}$ H. N. Phien, G. Vidal, and I. P. McCulloch, Phys. Rev. B 88, 035103 (2013).

${ }^{22}$ V. Zauner, M. Ganahl, H. G. Evertz, and T. Nishino, arXiv: 1207.0862 .

${ }^{23}$ W. Press, S. Teukolsky, W. Vetterling, and B. Flannery, in Numerical Recipes: The Art of Scientific Computing, 3rd ed. (Cambridge University Press, New York, 2007).

${ }^{24}$ C. Lubich and I. V. Oseledets, arXiv:1301.1058. 